ARTICLE

Received 27 Sep 2014 | Accepted 3 Apr 2015 | Published 11 May 2015

DOI: $10.1038 /$ ncomms 8097

OPEN

\title{
Structural basis for dynamic mechanism of nitrate/nitrite antiport by NarK
}

\author{
Masahiro Fukuda ${ }^{1,2, \star}$, Hironori Takeda ${ }^{1,2, \star}$, Hideaki E. Kato ${ }^{1,2, \dagger}$, Shintaro Doki ${ }^{1,2}$, Koichi Ito $^{3}$, Andrés D. Maturana ${ }^{4}$, \\ Ryuichiro Ishitani ${ }^{1,2} \&$ Osamu Nureki ${ }^{1,2}$
}

NarK belongs to the nitrate/nitrite porter (NNP) family in the major facilitator superfamily (MFS) and plays a central role in nitrate uptake across the membrane in diverse organisms, including archaea, bacteria, fungi and plants. Although previous studies provided insight into the overall structure and the substrate recognition of NarK, its molecular mechanism, including the driving force for nitrate transport, remained elusive. Here we demonstrate that NarK is a nitrate/nitrite antiporter, using an in vitro reconstituted system. Furthermore, we present the high-resolution crystal structures of NarK from Escherichia coli in the nitratebound occluded, nitrate-bound inward-open and apo inward-open states. The integrated structural, functional and computational analyses reveal the nitrate/nitrite antiport mechanism of NarK, in which substrate recognition is coupled to the transport cycle by the concomitant movement of the transmembrane helices and the key tyrosine and arginine residues in the substrate-binding site.

\footnotetext{
${ }^{1}$ Department of Biological Sciences, Graduate School of Science, The University of Tokyo, 7-3-1 Hongo, Bunkyo-ku, Tokyo 113-0033, Japan. ${ }^{2}$ Global Research Cluster, RIKEN, 2-1 Hirosawa, Wako-shi, Saitama 351-0198, Japan. ${ }^{3}$ Department of Medical Genome Sciences, Graduate School of Frontier Sciences, The University of Tokyo, 5-1-5 Kashiwanoha, Kashiwa-shi, Chiba 277-8562, Japan. ${ }^{4}$ Department of Bioengineering Sciences, Graduate School of Bioagricultural Sciences, Nagoya University, Furo-cho, Chikusa-ku, Nagoya 464-8601, Japan. ${ }^{\star}$ These authors contributed equally to this work. $†$ Present address: Department of Molecular and Cellular Physiology, Stanford University School of Medicine, Stanford, California 94305, USA. Correspondence and requests for materials should be addressed to R.I. (email: ishitani@bs.s.u-tokyo.ac.jp) or to O.N. (email: nureki@bs.s.u-tokyo.ac.jp).
} 
$\mathrm{N}$ itrogen is an essential element for all organisms and is present in numerous biological molecules, including DNA, RNA and protein. Although nitrogen is very abundant in the atmosphere, it is mostly present in the gaseous form, and therefore is not available to most organisms, except for diazotrophs $s^{1,2}$. Most organisms utilize nitrate, nitrite and ammonium ions as inorganic nitrogen sources. Nitrate respiration and assimilation are the principal processes in the nitrogen cycle of the ecosystem. In bacteria, nitrate is used as a substrate for anaerobic nitrate respiration ${ }^{3}$. Plants growing in aerobic soil absorb nitrate from the roots and assimilate it as a nutrient ${ }^{4}$. The first step of nitrate respiration and assimilation is cellular nitrate uptake, which is catalysed by nitrate transporter families; that is, the $\mathrm{ABC}$ transporter superfamily ${ }^{5}$, the Nitrate transporter 1/Peptide transporter Family (NPF) ${ }^{6,7}$ and the nitrate/nitrite porter (NNP) family. Although both the NNP and NPF families belong to the major facilitator superfamily (MFS), they share low amino-acid sequence similarity. The NNP family is the most prevalent nitrate transporter group conserved in archaea, bacteria, fungi, yeast, algae and plants ${ }^{8,9}$. The NNP family members in higher plants, such as NRT2.1 from Arabidopsis thaliana, are predominantly expressed in root cells, where they play important roles in nitrogen assimilation as key components of a high-affinity transport system for nitrate ${ }^{10}$. The bacterial NNP family transporters play an important role in the uptake of nitrate as a terminal electron acceptor for anaerobic respiration, and the extrusion of cytotoxic nitrite produced by anaerobic respiration ${ }^{11}$. In Escherichia coli, there are two NNP family transporters, NarK and NarU. NarK is highly expressed during anaerobic growth in the presence of nitrate, and plays a central role in nitrate uptake, while NarU has important functions during severe nutrient starvation or slow growth ${ }^{12}$. NarK and NarU share $75 \%$ amino-acid sequence similarity, suggesting that they have a similar transport mechanism.

Despite the importance of the NNP family transporters, their molecular-level functions are still under debate. Several hypotheses for the functions of NarK and NarU have been proposed: nitrate/nitrite antiporter ${ }^{13,14}$, nitrite $\mathrm{H}^{+}$antiporter ${ }^{11,15,16}$, nitrate $/ \mathrm{H}^{+}$symporter ${ }^{15-17}$, simple nitrite uniporter ${ }^{15,17}$ and $\mathrm{Na}^{+}{ }_{-}$and/or $\mathrm{K}^{+}$-coupled nitrate and/or nitrite transporter ${ }^{18}$. The lack of facile nitrogen and oxygen radioisotopes has hampered the direct measurement of their transport activity using in vitro reconstituted systems, and thus has limited an understanding of the precise functions of the NNP family transporters.

Recently, the crystal structures of NarK and NarU from E. coli were reported, at resolutions of $2.6-2.8$ and $3.0-3.1 \AA$, respectively $^{18,19}$. These structures revealed that both NarK and NarU contain 12 transmembrane (TM) helices with pseudo twofold symmetry axes, similar to other MFS transporters, and suggested that they transport substrates via an alternating-access mechanism ${ }^{20}$, in which the transporter adopts several conformations, involving outward-open, occluded and inwardopen states, during the transport cycle. Furthermore, these structures provided insights into the substrate recognition ${ }^{18,19}$. However, the molecular mechanism of the NNP family transporters remains largely unknown because of our poor understanding of their molecular-level functions and the lack of the atomic-resolution structures. Recently, the structures of another nitrate transporter, NRT1.1 from A. thaliana, in the NPF family, were reported ${ }^{21,22}$; however, its substrate recognition mechanism is entirely different from that of the NNP family, and thus the molecular mechanism of the NNP family remains elusive.

In this work, we demonstrate that NarK is a nitrate/nitrite antiporter, using an in vitro reconstituted system. Furthermore, to elucidate the precise molecular mechanism of nitrate/nitrite transport by NarK, we determined the crystal structures of NarK from $E$. coli in three different states (that is, nitrate-bound occluded, nitrate-bound inward-open and apo inward-open states) at $2.35-2.4 \AA$ resolutions. In combination with in vivo and in vitro functional analyses and molecular dynamics (MD) simulations, our results show the molecular mechanism of nitrate/nitrite antiport by NarK, in which the key tyrosine and arginine residues play essential roles in the coupling mechanism between the structural changes and nitrate/nitrite recognition.

\section{Results}

In vitro functional analysis of NarK. To understand the exact function of NarK, we developed an in vitro assay system using NarK-reconstituted proteoliposomes and the fluorescent probe, 4-amino-5-methylamino- $2^{\prime}, 7^{\prime}$-difluorescein $(\mathrm{DAF}-\mathrm{FM})^{23}$. Although DAF-FM was originally developed as a fluorescent probe for nitric oxide, it also reacts with nitrite under acidic conditions to form a fluorescent compound, triazolofluorescein, but not with other ions, including nitrate ${ }^{23}$ (Supplementary Fig. 1a,b). In this system, we quantified the amount of nitrite accumulated in the liposomes by measuring the fluorescence derived from the formation of triazolofluorescein. We observed the uptake of nitrite when nitrate was present inside the liposomes (Fig. 1a). In contrast, almost no nitrite uptake was observed when either acetate or propionate, which are larger than nitrate, was inside the liposomes (Fig. 1b). Anions with similar or smaller sizes than nitrate; that is, chloride, carbonate and formate, exhibited weaker nitrite-uptake activities than nitrate (Fig. 1b). While the lower $\mathrm{pH}$ resulted in increased transport activity in spite of the presence or absence of the membrane potential (Fig. 1c), the $\mathrm{pH}$ gradient across the membrane had no effect on the activity (Fig. 1d), suggesting that the $\mathrm{H}^{+}$concentration gradient across the membrane does not drive the substrate transport. Taken together, the flux of nitrite occurs in the presence of nitrate (and its near-cognate anions) as a counteranion, which is transported towards the outside of the liposomes. Although our results do not preclude the possibility that $\mathrm{NarK}$ is driven by $\mathrm{H}^{+}$, they clearly demonstrate that NarK is a nitrate/nitrite antiporter.

Overall structure. To elucidate the structural basis for the nitrate/nitrite antiport mechanism of NarK, we determined the crystal structures of NarK from E. coli. Three distinct types of crystals (I, II and III) were obtained, using the lipidic cubic phase (LCP) method $^{24}$. The phases were determined with the single isomorphous replacement with anomalous scattering (SIRAS) method, using the mercury-derivative crystal. We finally determined the crystal structures of NarK in three different states-the nitrate-bound occluded, nitrate-bound inward-open and apo inward-open states-at resolutions of 2.4, 2.4 and $2.35 \AA$, respectively (Table 1). Each asymmetric unit of Crystals I and II contained one inward-open molecule, while in the asymmetric unit of Crystal III, there were two occluded molecules (Mol A and B). Since Mol A and B have almost the same structure (r.m.s.d. of $0.38 \AA$ for all $\mathrm{C} \alpha$ atoms), we use Mol $\mathrm{A}$ in the following discussion. The overall structures of NarK in both the occluded and inward-open states adopt a canonical MFS-fold with 12 TM helices, with TM1-TM6 and TM7-TM12 forming the $\mathrm{N}$ and $\mathrm{C}$ bundles, respectively (Fig. 2a,b). These $\mathrm{N}$ and $\mathrm{C}$ bundles are connected with a linker loop between TM6 and TM7, which is disordered in the crystal structures (Fig. 2a,b). These overall structural features are consistent with the previously reported structures of $\mathrm{NarK}^{19}$ and $\mathrm{NarU}^{18}$. The substrate-binding site is located at the interface between the $\mathrm{N}$ and $\mathrm{C}$ bundles, 
a

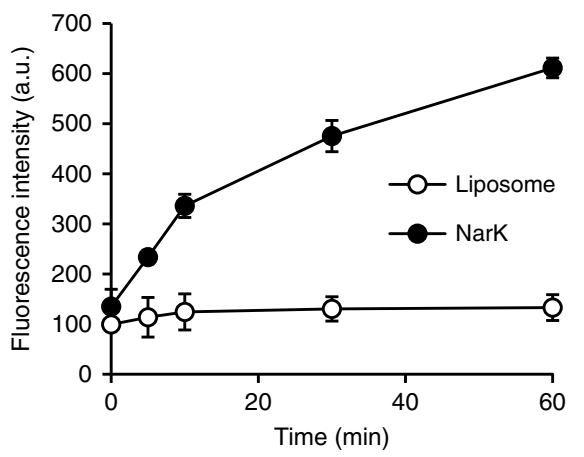

C

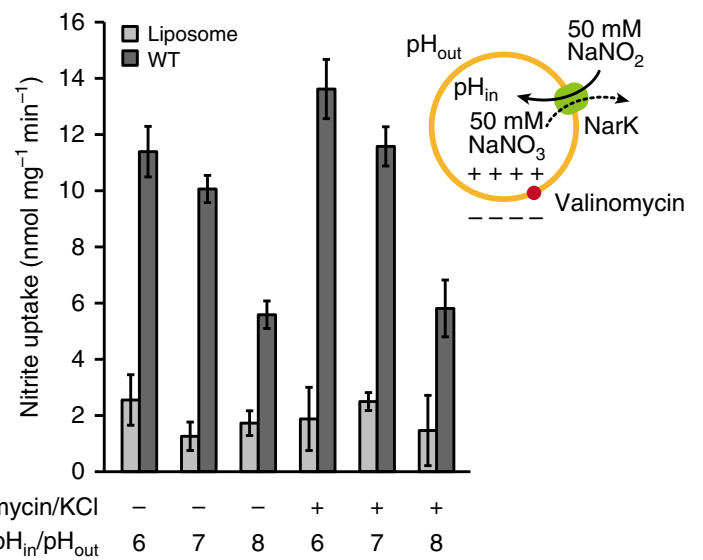

b

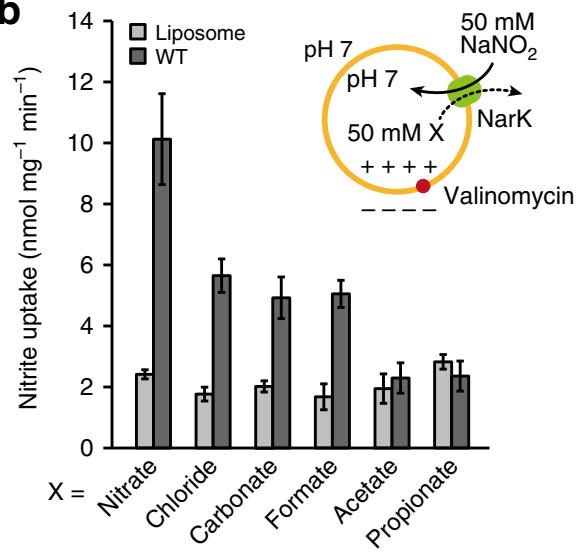

d

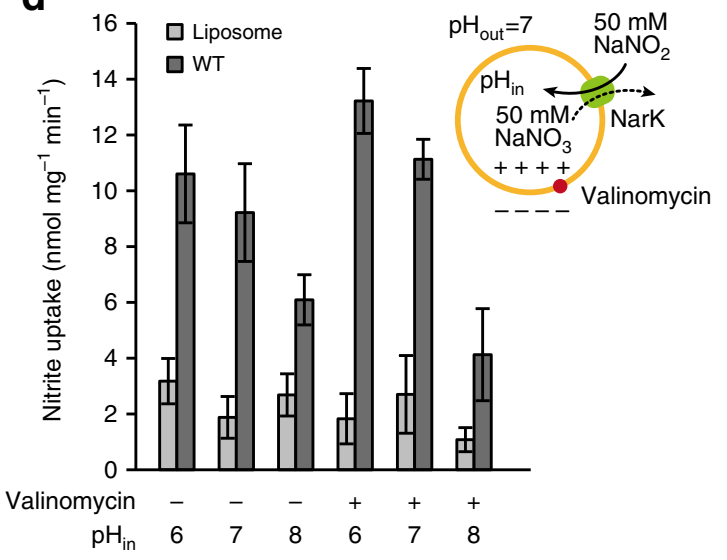

Figure 1 | Liposome-based nitrite-flux assay of NarK. (a) The time-dependent nitrite influx driven by the nitrate concentration gradient across the membrane at $\mathrm{pH} 7$, in the presence of a membrane potential, measured at $37^{\circ} \mathrm{C}$. The intra- and extraliposomal solutions contained $50 \mathrm{mM} \mathrm{NaNO} \mathrm{N}_{3}$ and $\mathrm{NaNO}_{2}$, respectively. (b) Substrate specificity of NarK. The intraliposomal solution contained $50 \mathrm{mM} \mathrm{NaNO}, \mathrm{NaCl}_{2} \mathrm{Na}_{2} \mathrm{CO}_{3}, \mathrm{HCOONa}, \mathrm{CH}_{3} \mathrm{COONa}_{3}$ $\mathrm{C}_{2} \mathrm{H}_{5} \mathrm{COONa}$. The extraliposomal solution contained $50 \mathrm{mM} \mathrm{NaNO}_{2}$ and was the same in all measurements. (c) pH and membrane-potential dependence of NarK activity. (d) Liposome-based nitrite-uptake assay in the presence of $\mathrm{pH}$ concentration gradients across the membrane. All error bars represent the s.d. of three independent trials.

in the middle of the TM segment (Fig. 2a,b). While the substrate-binding site is accessible from the cytoplasmic side in the inward-open structures, it is completely sequestered in the occluded state structure (Supplementary Fig. 2a-c).

Structural change of the TM helices in the $\mathrm{C}$ bundle. While no structural difference between the occluded and inward-open structures was observed in the $\mathrm{N}$ bundle, we observed a large structural change of the TM helices in the C bundle. The TM10 and TM11 helices are straight in the occluded structure, whereas they are bent around the conserved Gly residues (G363, G365, G367, G408, G414, G417 and G418) in the inward-open structures (Fig. 3a,b and Supplementary Fig. 3). Among them, G408, G414, G417 and G418 are included in the conserved Nitrate Signature motif 2 (refs 25,26). The cytosolic halves of TM10 and TM11 are rotated by 31 and 26 degrees, respectively, away from the centre of the transporter (Fig. 3a,b). Similar bending of TM10 and TM11 was also observed in the inward-facing NarK structures (PDB accession code: 4JR9) ${ }^{19}$; however, the TM10 and TM11 helices are further tilted towards the $\mathrm{N}$ bundle side in the present crystal structure (Supplementary Fig. 4a,b). The present crystal structures further reveal the structural change in TM7: as compared with the occluded state structure, the cytoplasmic half of TM7 in the inward-open state structure is rotated by 18 degrees towards the periphery, at the conserved G268 residue in the inward-open structure (Fig. 3a,b). As TM7 is located adjacent to TM10 and TM11 and forms tight interactions (Fig. 2a,b), the structural change of TM7 may be coupled with those of TM10 and TM11. G268 is strictly conserved among the NNP family transporters, including NarU from E. coli and NRT2.1 from $A$. thaliana (Supplementary Fig. 3), suggesting that a similar bending of TM7 occurs in other NNP transporters.

To confirm the importance of the conserved Gly residues in TM7, TM10 and TM11, we performed a genetic analysis of NarK mutants of these Gly residues. In this analysis, we examined the complementation activity of NarK mutants introduced by a plasmid, using a mutant E. coli strain lacking the operons of all nitrate and nitrite transporters, as well as two of the three nitrate reductases (BW25113 $\Delta$ nirC $\Delta$ narZ-narU $\Delta$ narK $\Delta$ napA-napG$n a p H-n a p B(\mathrm{DE} 3))^{15}$. Using the 'no-induction system', the complementation activities of NarK mutants were visualized by the colour of E. coli spots on MacConkey-glucose-nitrate-TMAO (trimethylamine $\mathrm{N}$-oxide) agar plates under anaerobic conditions $^{15}$ (see Methods). The results showed that the G408A and G418A mutants of TM11 and the triple Ala mutant of G363, G365 and G367 in TM10 ( $\Delta \mathrm{G} 3$ mutant) completely abolished the nitrate-uptake activity (Fig. 3c). These results suggested the importance of the flexibility of TM10 and TM11 conferred by these Gly residues. Furthermore, the G268A mutant of TM7 also completely abolished the nitrate-uptake activity (Fig. 3c), 


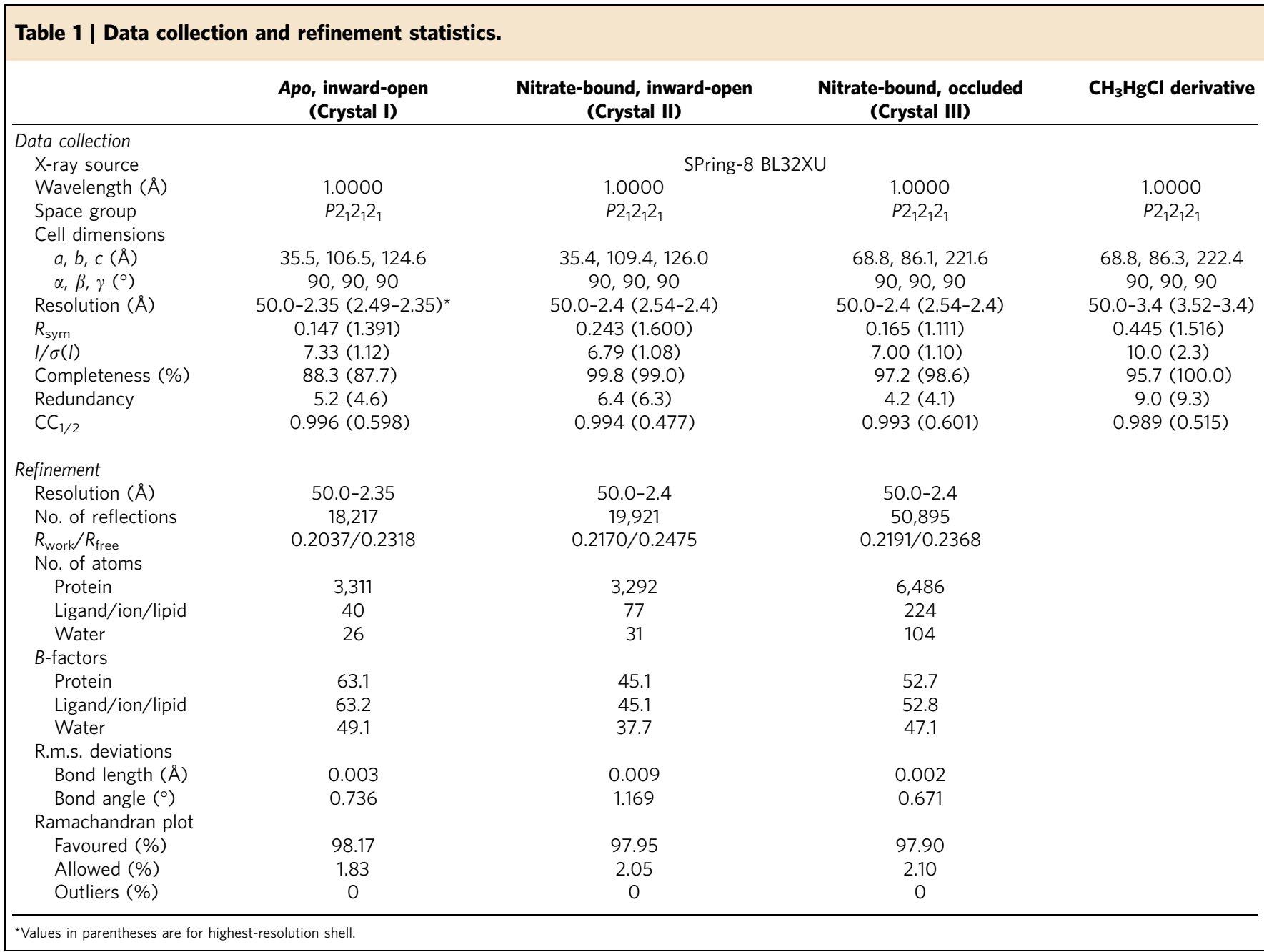

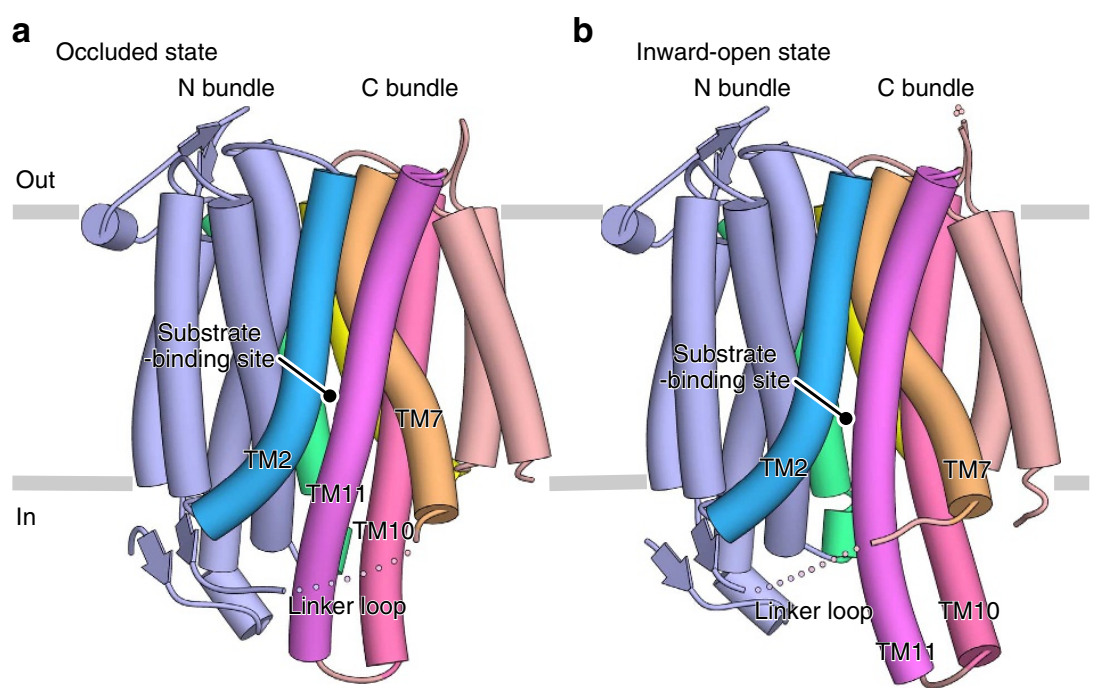

Figure 2 | Overall structure of E. coli NarK. (a,b) Overall structures of NarK in the occluded (a) and inward-open (b) states. Each molecule contains 12 TM helices, forming the N bundle (TM1-6) and the C bundle (TM7-12). TM2, TM5, TM7, TM8, TM10 and TM11 are coloured light blue, green, amber, yellow, pink and purple, respectively. Other TM helices in the $\mathrm{N}$ and $\mathrm{C}$ bundles are coloured pale blue and light pink, respectively. Grey bars indicate the approximate location of the lipid bilayer. The figures depicting the molecular structures were prepared using CueMol (http://www.cuemol.org/).

suggesting that the bending of TM7 around the G268 residue is critical for the nitrate transport activity by NarK.

Although no structural difference was observed in the other TM helices (that is, TM2, TM4, TM5 and TM8), they also include many conserved Gly residues (Supplementary Fig. 3). Especially, G164, G165, G168, G171, G172, G174 and G177 in TM5 are included in Nitrate Signature motif 1 (refs 25,26). To investigate the importance of these Gly residues, we performed the genetic 
a
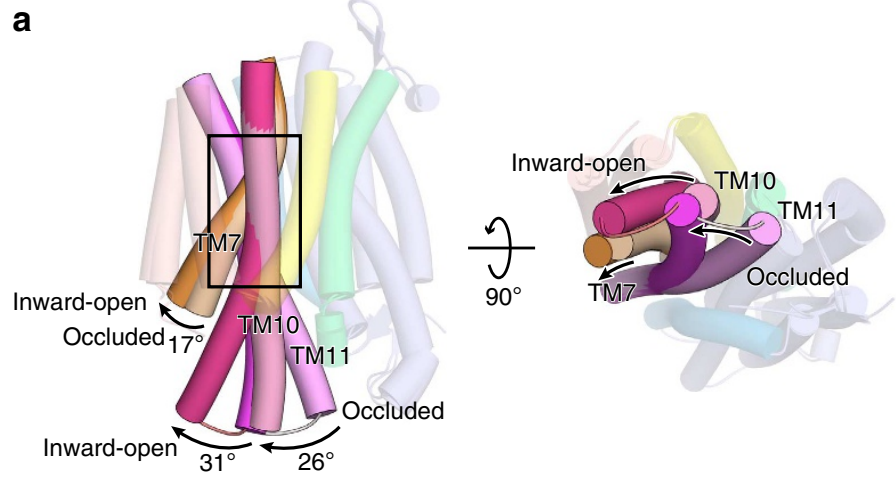

b

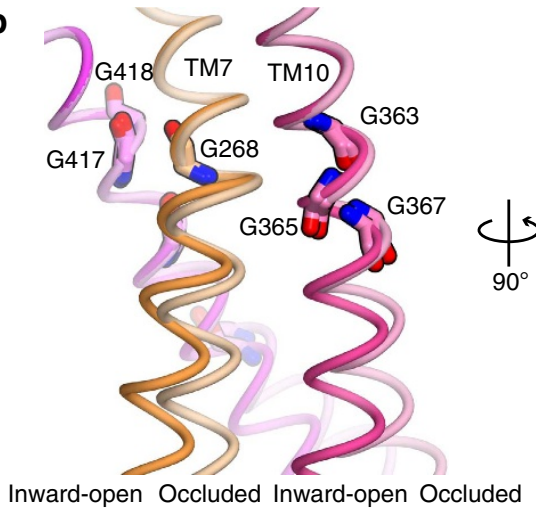

C
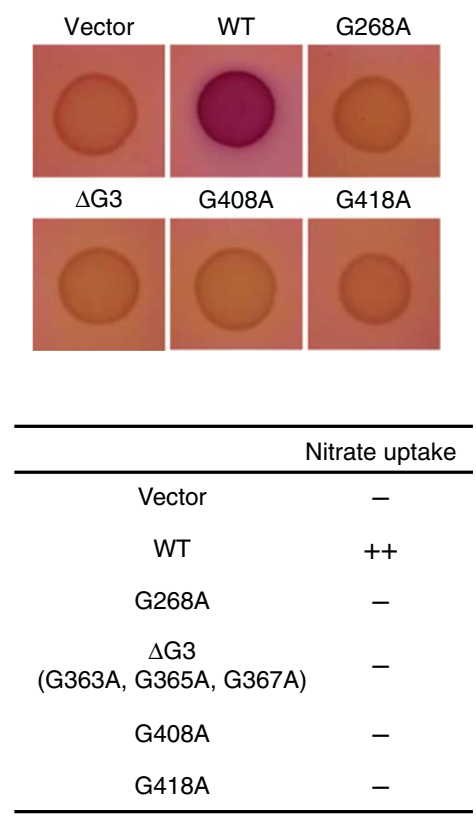

Figure 3 | Structural comparison of the occluded and inward-open states. (a) Overall structures of NarK in the occluded and inward-open states, viewed from the plane of the membrane (left panel) and the cytoplasmic side (right panel). The two structures were superimposed based on the C $\alpha$ atoms of the $\mathrm{N}$ bundle residues (1-233). The colours of the helices in the inward-open state correspond to those in Fig. 2a,b. TM7, TM10 and TM11 in the occluded state are depicted with pale colours. The rectangle indicates the region highlighted in b. (b) Close-up views around the conserved Gly residues in TM7, TM10 and TM11. The occluded and inward-open structures were superimposed as in $\mathbf{a}$. The Gly residues are shown in stick models. (c) Genetic analysis of the nitrate transport activity of NarK mutants of the conserved Gly residues involved in the bending of TM7, TM10 and TM11, using the no-induction system. E. coli cultures were spotted on MacConley-glucose-TMAO agar plates under anaerobic conditions, and their colours were monitored. E. coli cells expressing intact NarK accumulate nitrate, generating dark-red spots, while those expressing inactive NarK generate pale-yellow spots (see Methods).

The results of this genetic analysis are summarized in the table on the right.

analysis of their mutants. The results also indicated the importance of the Gly residues, G85 in TM2, G141 and G144 in TM4, G164, G168, G172 and G177 in TM5, and G308 and G309 in TM8, for the nitrate-uptake activity (Supplementary Fig. 5).

Structural change in the transport path. In the crystal structure of the nitrate-bound occluded state, both the cytosolic and periplasmic sides are tightly closed by hydrophilic and hydrophobic interactions between the $\mathrm{N}$ and $\mathrm{C}$ bundles (Fig. 4a and Supplementary Fig. 6a,b), and many of these interactions were not observed in the NarU structure previously reported as the occluded state (PDB accession code: 4IU8, Mol A $)^{18}$ (Supplementary Fig. 4c,d). Furthermore, the present highresolution crystal structures reveal the atomic details of these interactions, including water-mediated interactions (Supplementary Fig. 4c,d and Supplementary Fig. 6b). In the cytoplasmic pathway, F370 and L407 in the C bundle hydrophobically interact with F147, M151 and L167 in the N bundle, forming the interaction layer $\mathrm{C} 1$ (Fig. 4a). Furthermore, the main-chain carbonyl groups of A404 in TM11 and A148 in TM4 form a water-mediated hydrogen bond (Fig. 4a). These interactions in layer $\mathrm{C} 1$ occlude the pathway from the substratebinding site towards the cytosolic side. In addition to these hydrophobic interactions, TM4 and TM5 in the N bundle form a number of hydrophobic and hydrophilic interactions with TM10 and TM11 in the $\mathrm{C}$ bundle, thereby closing the transport pathway (layers C2 and C3; Fig. 4a). In layer C2, the side chain of F156 in TM4 and the main chain of P159, K160 and Q161, in the loop between TM4 and TM5, form hydrophobic interactions with the side chains of M382 in TM10 and M396 and A400 in TM11 (Fig. 4a). The guanidinium group of R378 in TM10 hydrogen bonds with the main-chain carbonyl groups of F158 and K160, and the main-chain carbonyl group of A400 hydrogen bonds with the hydroxyl group of S155 in TM4 (Fig. 4a and Supplementary Fig. 7a). In layer C3, part of the N-terminal loop consisting of P20 and E21 forms van der Waals contacts with the cytosolic terminus of TM11, including D392, E393 and M396 (Fig. 4a). These interactions between the $\mathrm{N}$ and $\mathrm{C}$ bundles tightly close the pathway to the cytoplasmic side to prevent the intrusion of water or other solvent molecules into the substrate-binding site. In the structure of NarU, the cytoplasmic pathway is reportedly closed by a thin gate consisting of only two residues, F145 and F367 (corresponding to F147 and F370 in NarK, respectively), and the extensive interactions involving TM4 and TM5 in the $\mathrm{N}$ bundle and TM10 and TM11 in the $C$ bundle are missing ${ }^{18}$ (Supplementary Fig. 4d). However, given that several residues of NarK involved in the above interactions, including R378, are conserved in NarU (Supplementary Fig. 3), the cytoplasmic side of NarU would be further closed by the interactions involving TM4, TM5, TM10 and TM11 in the occluded state. Therefore, the 
a

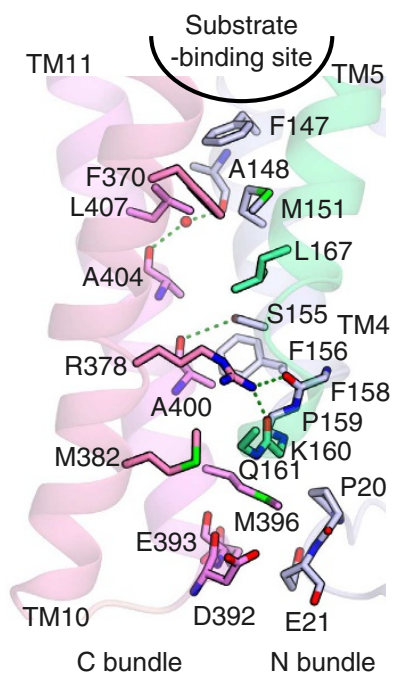

b

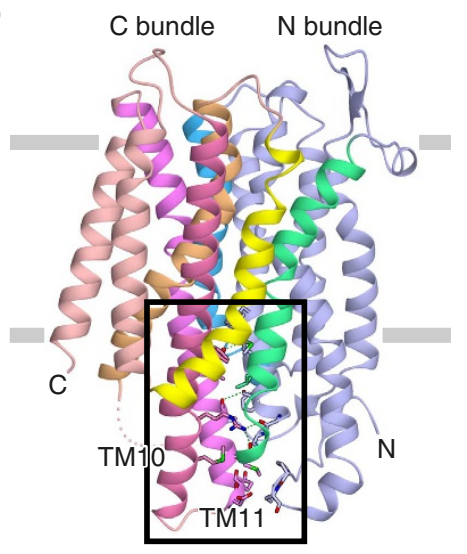

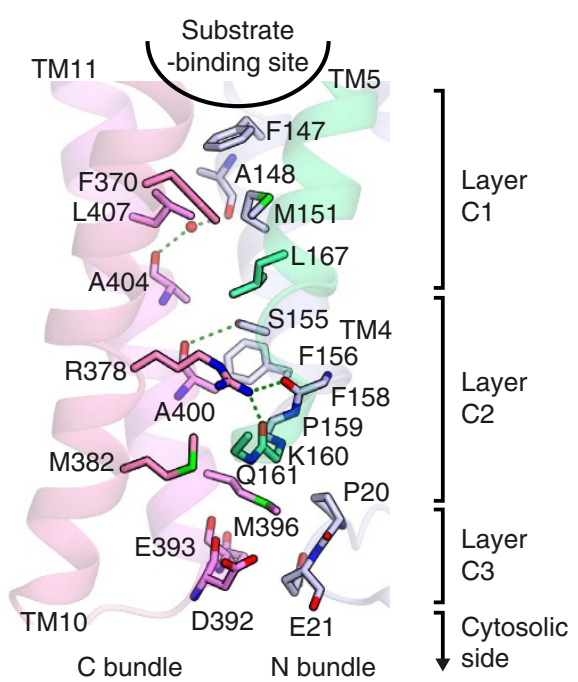

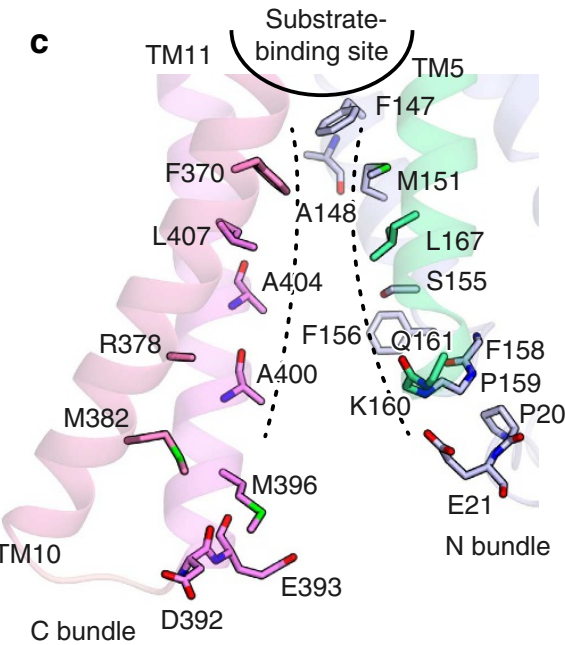

Figure 4 | The structural change in the cytosolic transport pathway of NarK. (a) The cytoplasmic interactions between the N and C bundles of the occluded state (stereo view). Residues related to the interactions are depicted with stick models. The side chains from F158 to Q161 are omitted for clarification. Water molecules are represented by red spheres. The green dashed lines indicate hydrogen bonds. The area highlighted in this panel is indicated by the rectangle in the overall structure shown in b. (b) The overall structure of NarK in the nitrate-bound occluded state. The side chains involved in the cytoplasmic interactions are depicted by stick models. (c) The cytoplasmic area between the $\mathrm{N}$ and $\mathrm{C}$ bundles of the inward-open state. The black dashed lines indicate the transport pathway observed in the inward-open state, which is closed in the occluded state.

previously reported occluded state structure of $\mathrm{NarU}^{18}$ may actually represent the partially occluded inward-facing state (Supplementary Fig. 4a,b).

In contrast to their conformations in the present occluded structure of NarK, in the inward-open structure, TM7, TM10 and TM11 in the $\mathrm{C}$ bundle are bent at the conserved Gly residues (Fig. 3b). As a result, none of the interactions in layers C1, C2 and C3 is formed (Fig. 4b). The transport pathway is thereby formed between the $\mathrm{N}$ and $\mathrm{C}$ bundles, and the substrate-binding site is accessible from the cytosolic side (Fig. $4 \mathrm{~b}$ ). The previous structure of NarK bound to the antibody Fab fragment is reported in the inward-facing conformation (PDB accession code: 4JR9) ${ }^{19}$. However, the hydrophobic interactions between F147, M151 and L407 are still formed, and thus the substrate-binding site is not accessible in the previous structure (Supplementary Fig. 4a,b). Therefore, the previously reported structure of NarK is still in the intermediate, partially occluded inward-facing state. In contrast, TM10 and TM11 in the present inward-open structure are further rotated towards the periphery, thereby completely opening the cytoplasmic pathway (Fig. 4c and Supplementary Fig. 4a,b).
Nitrate recognition in the substrate-binding site. In the nitratebound occluded structure, we observed a strong electron-density peak in the substrate-binding site (Fig. 5a). This flat, triangular density peak was present in a location consistent with those of the bound nitrate and nitrite ions in the $\mathrm{NarU}^{18}$ and $\mathrm{NarK}^{18}$ structures, respectively. The crystallization conditions for the occluded-state crystals (Crystal III) contained ammonium nitrate. Therefore, we assigned this peak as a nitrate ion. The present crystal structures have clarified the interactions between the substrate and the protein side chains (Fig. 5a), as compared with the previous crystal structures of $\mathrm{NarU}^{18}$ and $\mathrm{NarK}^{18}$. The substrate-binding site is mainly formed by the side chains of F49, R89, F147, N175, Y263, F267 and R305 (Fig. 5a and Supplementary Fig. 8a,b), which are highly conserved among the NNP family transporters (Supplementary Fig. 3). The nitrate ion is sandwiched between F147 and F267, and forms a $\pi-\pi$ stacking interaction with the $\pi$ electrons of their phenyl groups (Fig. $5 \mathrm{a}$ and Supplementary Fig. 8a,b). The oxygen atoms of the nitrate ion are further recognized by three sites: A, B and C (Fig. 5a and Supplementary Fig. 8a,b). In Site A, two oxygen atoms of the 

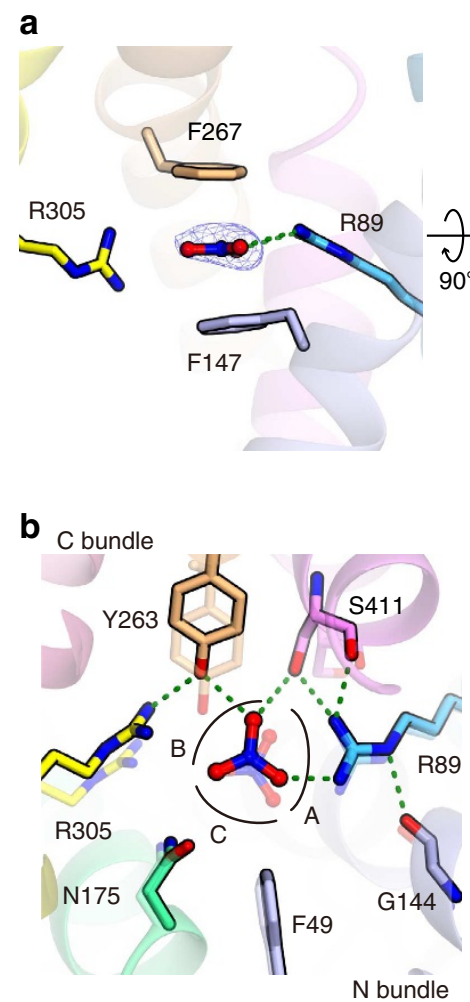

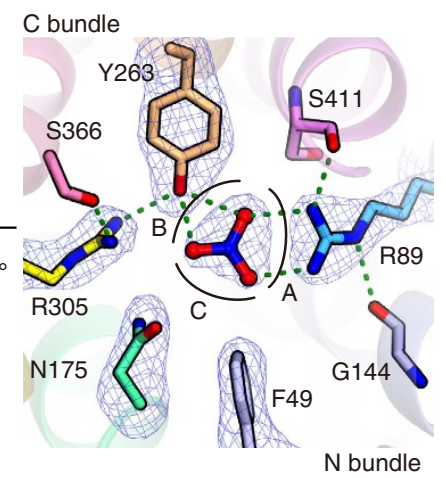

d

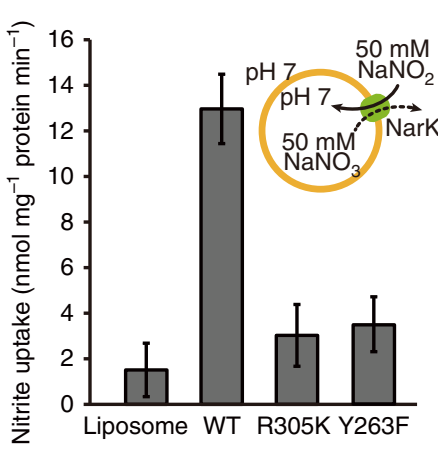

C
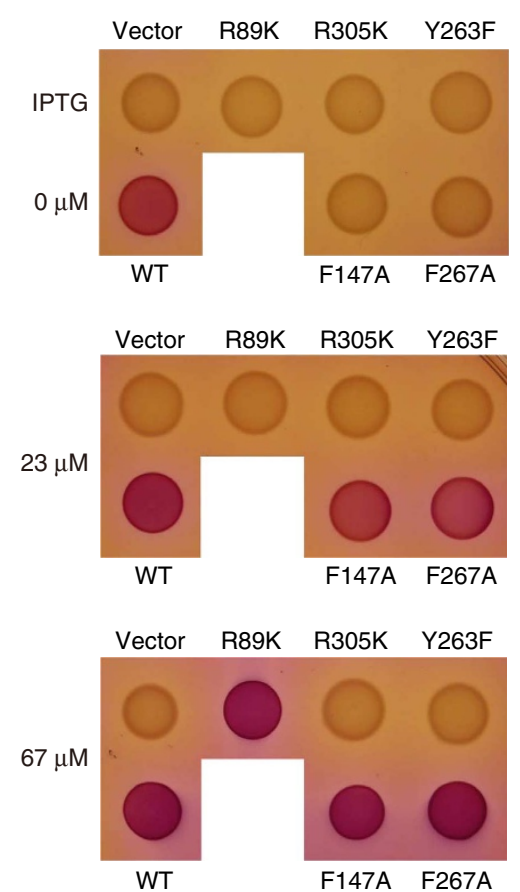

Figure 5 | The nitrate recognition mechanism and the structural change in the substrate-binding site of NarK. (a) Close-up views of the substratebinding site in the nitrate-bound occluded state $(\mathrm{Mol} A)$, viewed from two different directions. The electron-density map of the $m F_{\mathrm{o}}-D F_{\mathrm{c}}$ omit maps, contoured at $4 \sigma$ for nitrate ion (left panel) and nitrate ion, F49, R89, N175, Y263 and R305 (right panel), respectively. The colours of the carbon atoms represent the TM helices with the same colour scheme as in Fig. 2a,b. The green dashed lines indicate the hydrogen bonds up to $3.1 \AA$. (b) Close-up view of the substrate-binding site in the nitrate-bound inward-open state. The residues in the occluded state are semitransparent, for comparison. (c) Genetic analysis of the nitrate transport activity of NarK mutants directly involved in substrate recognition, using the inducible system. The results are summarized in the table on the right. (d) Liposome-based nitrite-flux assay of NarK mutants (Y263F and R305K). All error bars represent the s.d. of three independent trials.

nitrate ion form a bidentate salt bridge with the $\mathrm{NH} 1$ and $\mathrm{NH} 2$ atoms of the R89 guanidinium group. This guanidinium group of R89 is anchored by the main-chain carbonyl groups of G144 in TM4 and S411 in TM11, and together they form a rigid substratebinding site (Fig. 5a and Supplementary Fig. 8a,b). In Site B, two oxygen atoms of the nitrate ion form a bifurcated hydrogen bond with the phenol hydroxyl group of Y263 in TM7. This hydroxyl group hydrogen bonds with the R305 guanidinium group, which in turn hydrogen bonds with the S366 hydroxyl group (Fig. 5a and Supplementary Fig. 8a). As a result, the guanidinium group of R305 is oriented perpendicularly to the substrate nitrate ion. In Site $\mathrm{C}$, the side-chain amide group of N175 and the phenyl group of F49 form an interaction site for the nitrate ion (Fig. 5a and Supplementary Fig. 8a,b). Overall, these conserved amino-acid residues create a suitable substrate-binding site for a flat anion with delocalized $\pi$ electrons.

Similar nitrate/nitrite recognition by Arg side chain and/or aromatic groups is also observed in several proteins. For example, in molybdenum-containing $\mathrm{NAD}(\mathrm{P}) \mathrm{H}$ :nitrate reductase, the nitrate ion is reportedly recognized by two Arg residues ${ }^{27}$. In contrast, the manner of nitrate recognition by the other nitrate transporter, NRT1.1, is quite different from that of NarK, as NRT1.1 recognizes nitrate by Thr and His residues ${ }^{21,22}$.

In the nitrate-bound, inward-open structure, a triangular electron-density peak of a nitrate ion was also observed in the substrate-binding site (Supplementary Fig. 7b). However, we found several differences in the recognition manner of the substrate nitrate, as compared with that in the occluded state.
In the inward-open structure, the bending of TM7, TM10 and TM11 leads to a slight movement of the side chains of Y263 in TM7 and R305 in TM8, away from the centre of the transporter (Fig. 5b). As a result, the following changes were observed in the interactions in Sites A and B. In Site A, the distance between one oxygen atom of the nitrate ion and the R89 guanidinium group is increased from 2.7 to $3.1 \AA$ (Fig. 5b and Supplementary Fig. 8a,c). In Site B, the distance between the nitrate ion and the Y263 phenol group is increased from 2.4 to $3.9 \AA$, and only one oxygen atom is within hydrogen-bonding distance with the Y263 phenol group (Fig. 5b and Supplementary Fig. 8a,c). These changes in Sites A and B increase the volume of the substrate-binding site in the inward-open state structure $\left(37.6\right.$ and $50.5 \AA^{3}$ for the occluded and inward-open structures, respectively; Supplementary Fig. 9a,b). Taken together, the overall structural change in the transition from the nitrate-bound occluded to the inward-open state, including the bending of TM7, TM10 and TM11, induces the movement of Y263 and R305 in Site B, which enlarges the volume of the substrate-binding site. This change in the substrate-binding site may result in decreased substrate affinity in the inward-open state, thereby facilitating substrate release towards the cytoplasmic side.

In contrast, the apo inward-open structure was similar to the nitrate-bound, inward-open structure (r.m.s.d. of $0.51 \AA$ for $435 \mathrm{C} \alpha$ atoms; Supplementary Fig. 8c,d and Supplementary Fig. 10a,b). While no electron-density peak was observed in the substrate-binding site in the apo structure of the inward-open state (Supplementary Fig. 7c), the positions of the side chain 
atoms in the substrate-binding site are superimposable on those of the nitrate-bound structure (Supplementary Fig. 10b). This suggested that the inward-open state is also stable without the substrate, and that the binding and release of the substrate can occur without any large structural changes.

Mutational analysis of the substrate recognition residues. To examine the importance of the residues involved in the substrate recognition, we performed the structure-based genetic analysis of NarK mutants using the 'inducible system' (see Methods). The results showed that the Ala mutants of both F147 and F267, which form $\pi-\pi$ interactions with nitrate (Fig. $5 \mathrm{a}$ ), did not restore the nitrate-transport activity to the same level as that of wild-type NarK (Fig. 5c), while complementation was observed with higher isopropyl- $\beta$-D-thiogalactoside (IPTG) concentrations (23 and $67 \mu \mathrm{M}$; Fig. 5c). This result suggested that the $\pi-\pi$ stacking interaction between the substrate nitrate and the phenyl groups of F147 and F267 is important, but not critical for the transport activity. The R89K mutant also abolished the complementation activity, but rescued the nitrate-uptake activity under the conditions with the highest IPTG concentration $(67 \mu \mathrm{M}$; Fig. $5 \mathrm{c})$. This result suggested that the positive charge at the R89 position is important for the substrate recognition. In contrast, the Y263F and $\mathrm{R} 305 \mathrm{~K}$ mutants completely abolished the complementation activity, even under the conditions with the higher IPTG concentrations (23 and $67 \mu \mathrm{M}$; Fig. 5c). The results obtained with the Y263F mutant suggested that the phenol hydroxyl group of Y263, which hydrogen bonds with both the substrate nitrate and R305 guanidinium group, is critical for the transport activity. In addition, the results from the R305K mutant suggested that the guanidinium group of R305 itself, rather than simply a positive charge, is essential for the transport activity. Furthermore, the purified Y263F and R305K mutants showed no transport activity in our reconstituted system (Fig. 5d). The results from these genetic and in vitro analyses support our proposal that Y263 and R305 in the substrate-binding site are the key residues for coupling the bending of the TM helices with the substrate recognition.

MD simulation. To clarify the dynamic coupling mechanism between the state transition and the substrate recognition, we performed MD simulations based on the present crystal structure. In each of three independent simulations starting from the occluded state with the bound nitrate, no large structural change in the overall conformation was observed during the 250-ns simulation (Fig. 6a and Supplementary Fig. 11a,b), suggesting that the overall structure of the occluded state is stable in the lipid bilayer environment. The structure of the substrate-binding site, including the nitrate recognition manner, remained unchanged during the simulation (Supplementary Fig. 12a), showing that the substrate-binding site of the occluded state structure is stable with the bound nitrate.

Next, to examine the effect of the nitrate-binding and release on the occluded state structure, we performed the three independent simulations starting from the occluded state without the bound nitrate. Interestingly, a large structural change was observed in the first $10 \mathrm{~ns}$ (Fig. 6a), mainly in the TM10 and TM11 helices in the $\mathrm{C}$ bundle (Fig. $6 \mathrm{~b}$ ). The interaction between TM4-TM5 and TM10-TM11 was lost in the first 10 ns (Fig. 6c), including the hydrogen bonds between R378 and the loop connecting TM4 and TM5 (Supplementary Fig. 12b). Similar structural changes were reproducibly observed in the other two simulations (Supplementary Fig. 11c,d). The transport path from the cytosolic side to the substrate-binding site opened, and water molecules entered the substrate-binding site (Supplementary
Fig. 13a,b). After $250 \mathrm{~ns}$ in the simulation, the distance between TM4-TM5 and TM10-TM11 was increased to a similar level as that in the inward-open structure (Fig. 6c,d). In addition, we also performed the MD simulation starting from the apo inward-open structure. The result showed that the apo inward-open structure is stable, and no structural change towards the occluded state was observed without any substrates during the 250-ns simulation (Supplementary Fig. 14).

Taken together, these results suggested that the negatively charged nitrate ion, bound between the positively charged R89 and R305 residues, relaxes the electrostatic repulsion between them, thereby facilitating the formation of the occluded state. In contrast, without the substrate nitrate ion, the electrostatic repulsion between $\mathrm{R} 89$ and $\mathrm{R} 305$ allows the $\mathrm{N}$ and $\mathrm{C}$ bundles to segregate from each other, thereby facilitating the formation of the inward-open state.

\section{Discussion}

The locations of the nitrate ions found in the present NarK structures are consistent with those of the nitrite ion in the previous crystal structure of $\mathrm{NarK}^{19}$, suggesting that both nitrate and nitrite bind to the same substrate-binding site competitively. The present atomic-resolution structures of the nitrate-bound NarK provided further structural insights into the nitrite ion recognition mechanism (Supplementary Fig. 15a). Given that nitrite also has a negative charge and delocalized $\pi$ electrons, it can bind to the substrate-binding site between the phenyl groups of F147 and F267, as observed in the nitrate-bound structures. The interactions in Sites A and B are also possible, in the case of nitrite. The nitrite oxygen atoms can form a bidentate salt bridge with the $\mathrm{NH} 1$ and $\mathrm{NH} 2$ atoms of R89, and hydrogen bond with the hydroxyl group of Y263 (Supplementary Fig. 15a), as in the nitrate-bound structures (Fig. 5a). The nitrogen atom of nitrite has lone-pair electrons, and thus it could further hydrogen bond with the hydroxyl group of Y263 (Supplementary Fig. 15a). In contrast, nitrite has only two oxygen atoms, and is smaller than nitrate. Therefore, the interactions involving one of the three oxygen atoms of nitrate are impossible in the case of nitrite. In our nitrite-bound model, the side-chain amide group of N175 (that is, Site C) is not involved in substrate recognition (Supplementary Fig. 15a). This notion is consistent with the results from a previous study, showing that the affinity of NarU for nitrite is $\sim 10$-fold lower than that for nitrate ${ }^{18}$

The present in vitro functional analysis revealed that chloride ions weakly facilitate the nitrate-uptake activity (Fig. 1b), suggesting that the chloride ion binds to the substrate-binding site to be transported as a counteranion. Although the chloride ion lacks $\pi$ electrons, it can bind between the R89 and R305 residues to induce the formation of the occluded state. This is consistent with the results of the genetic analysis of F147 and F267, showing that the $\pi-\pi$ stacking interaction with the substrate is important, but not critical for the transport activity. The results of the in vitro functional analysis also showed that carbonate and formate ions weakly facilitate the nitrate-uptake activity (Fig. 1b). Given that carbonate ion is singly protonated to form bicarbonate ion under the assay conditions, the proton of the bicarbonate cannot fit within the substrate-binding site (Supplementary Fig. 15b). This may reduce the affinity of the bicarbonate ion to the substrate-binding site, as compared with that of the nitrate ion, despite their similar molecular shapes. The formate ion has a hydrogen atom bound to the carbon atom, while the nitrite ion has lone pair electrons at the corresponding nitrogen atom. Thus, the formate ion also cannot fit within the substrate-binding site, although it has a similar molecular shape 

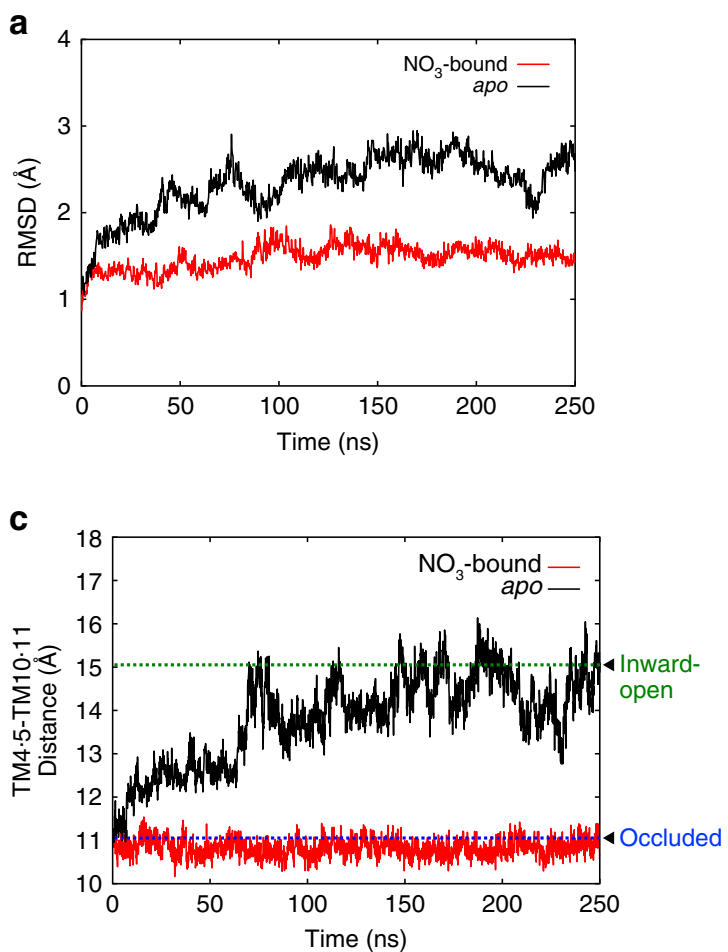
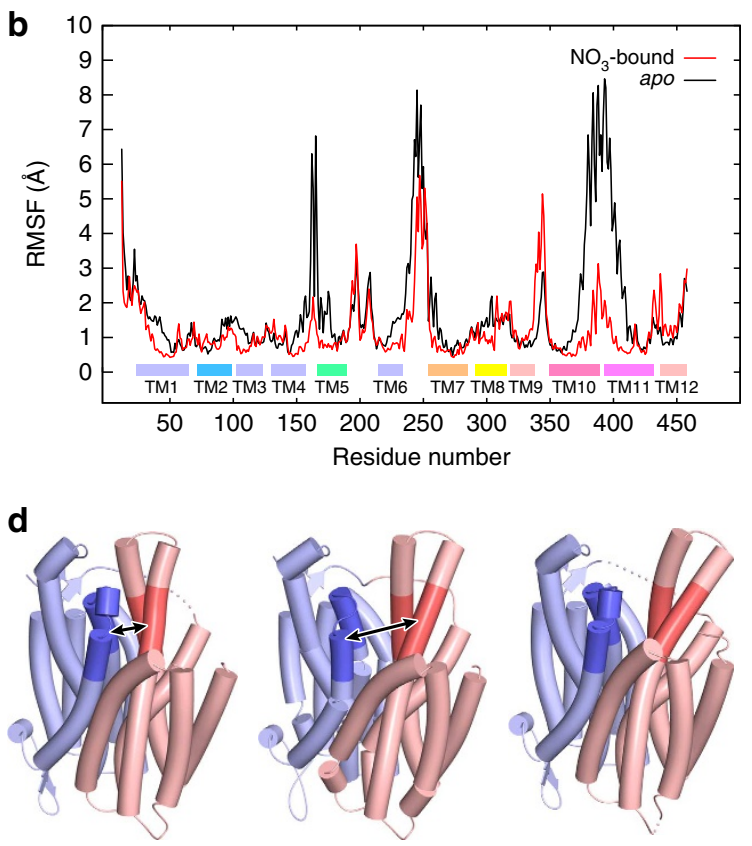

Initial structure
(occluded)

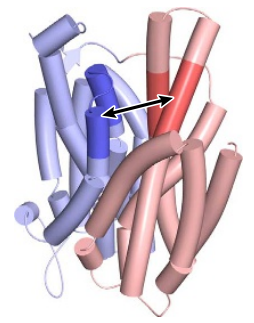

MD snapshot (at $250 \mathrm{~ns}$ )

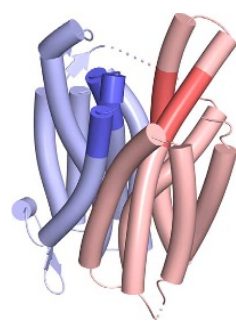

Crystal structure (inward-open)

Figure 6 | Molecular dynamics simulations of NarK. (a,b) Structural deviations and fluctuations observed in the molecular dynamics simulations. (a) Plots of the RMS deviations of the overall $\mathrm{C} \alpha$ atoms from the initial crystal structures as a function of time, during the nitrate-bound and apo occluded simulations. (b) Plots of the time-averaged RMS fluctuations of each residue from the initial crystal structures, during the nitrate-bound and apo occluded simulations. The locations of the TM segments are indicated by the boxes, which are coloured as in Fig. 2a,b. (c) Plots of the distances between the centroids of the $\mathrm{C} \alpha$ atoms of the cytosolic parts of TM4-TM5 (A148 to G171) and TM10-TM11 (T369 to L380 and A399 to F409), as a function of time, during the nitrate-bound and apo occluded simulations. The distances between the centroids of the C $\alpha$ atoms of the cytosolic parts of TM4-TM5 and TM10-TM11 of two crystal structures (occluded and inward-open state) are shown by blue and green dashed lines, respectively, and indicated by black rectangles. (d) The initial structure, the snapshot structure of the apo simulation at $250 \mathrm{~ns}$, and the crystal structure in the inward-open state are shown. The $\mathrm{N}$ and $\mathrm{C}$ bundles are coloured pale blue and pink, respectively. The regions in TM4, TM5, TM10 and TM11 used for the distance measurement are highlighted in deep blue and red colours and are indicated by black arrows.

as the nitrite ion (Supplementary Fig. 15c). In contrast, the present in vitro analysis showed that acetate and propionate ions do not facilitate the nitrate-uptake activity (Fig. 1b). The bulky methyl and ethyl groups may completely prevent the interaction of these anions with the substrate-binding site, and thus they are not transported as counteranions. These results with the acetate and propionate ions also suggested that NarK is a tightly coupled antiporter, which does not transport substrates in the absence of counteranions.

In conclusion, we demonstrated that NarK is a nitrate/nitrite antiporter, by an in vitro functional analysis. This conclusion is also consistent with the results of MD simulations with and without nitrate in the substrate-binding site. Under physiological conditions, it is reasonable that NarK functions as a system for both the uptake of nitrate, as an electron acceptor for anaerobic respiration and the extrusion of cytotoxic nitrite produced by anaerobic respiration. The present crystal structures and structure-based genetic analyses have elegantly explained the coupling mechanism between the substrate recognition and the structural changes that underlie the nitrate/nitrite antiport mechanism. Our working model of nitrate/nitrite antiport by NarK explains the half of the transport cycle involving the inward-open state. In the nitrate-bound occluded state, the negative charge of the nitrate in the substrate-binding site relaxes the electrostatic repulsion between the two positively charged arginine residues (R89 and R305), thereby enabling these two arginine residues to be in close proximity in the low-dielectric intramembrane environment (Fig. 7). The following structural change to the inward-open state induces the bending of the helices, TM7, TM10 and TM11, around the conserved Gly residues, which opens up the transport pathway from the substrate-binding site to the cytoplasmic side between the $\mathrm{N}$ and $\mathrm{C}$ bundles (Fig. 7 and Supplementary Movie). These bending motions of the TM helices are coupled with the movement of the side chains of Y263 in TM7 and R305 in TM8 away from the $\mathrm{N}$-bundle side, which enlarges the volume of the substratebinding site and decreases the affinity of NarK for nitrate, thereby facilitating nitrate release to the cytoplasm. The key residues for this coupling mechanism (Y263, R305 and G268) are strictly conserved in the NNP family (Supplementary Fig. 3), suggesting that other NNP family members, including NRT2.1 of A. thaliana, transport nitrate ions in a similar manner. The electrostatic repulsion between the positive charges of R89 and R305 prohibits the movement of Y263 and R305 towards the $\mathrm{N}$-bundle side, thus preventing the closure of the transport pathway by the movement of TM7, TM10 and TM11 of the C bundle towards the N-bundle side (Fig. 7 and Supplementary Movie). In the next step, a nitrite ion in the cytoplasm is bound to the substrate-binding site in a similar manner to that of nitrate (Supplementary Fig. 15a), which may facilitate the conformational change to the outward-open state via the nitrite-bound occluded state. In this structural change between the occluded and outward-open states, the conserved Gly residues in TM2, TM4, TM5 and TM8 may play important roles in the conformational flexibility, as suggested by our genetic analysis (Supplementary Fig. 5). Further understanding of the remaining 


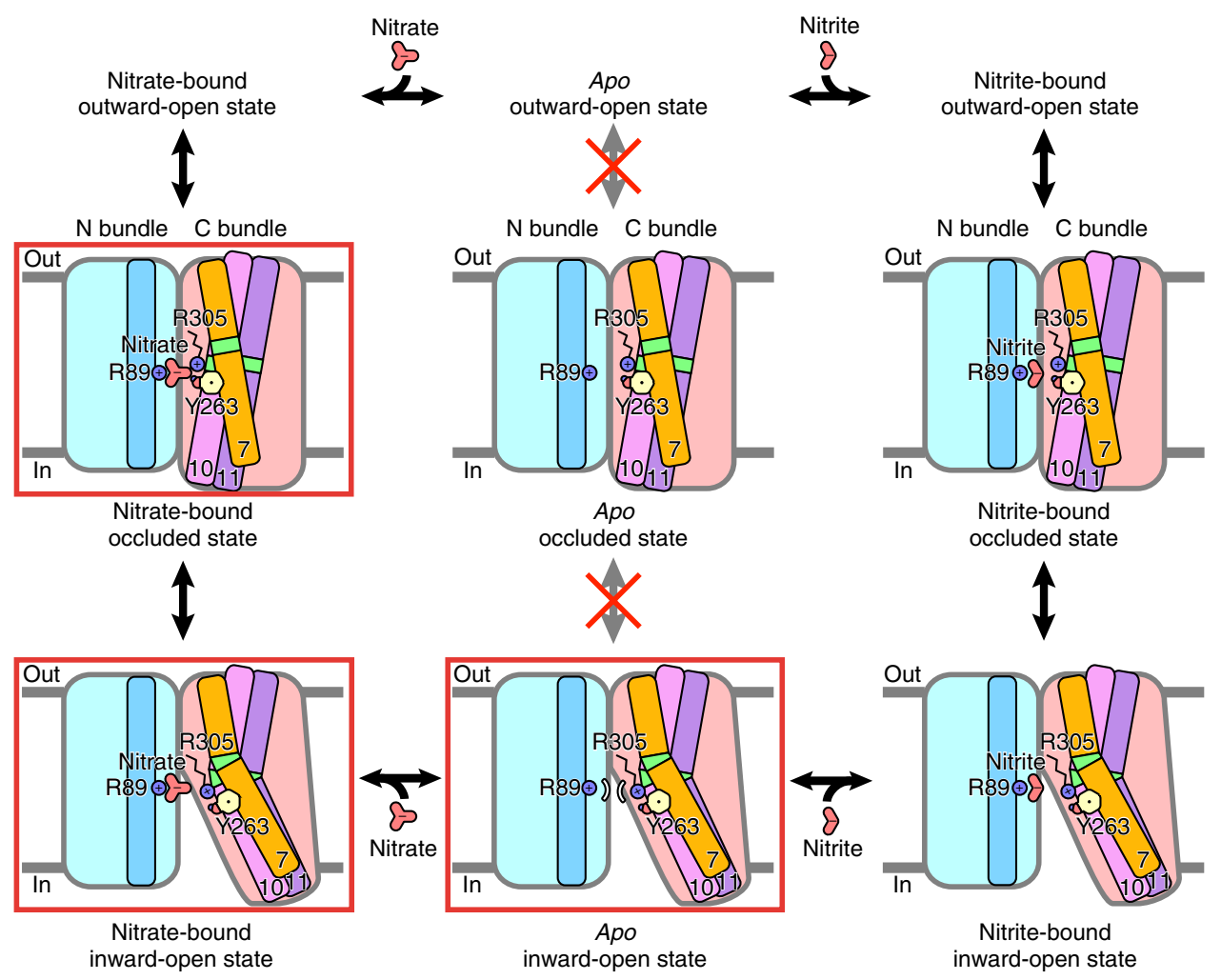

Figure 7 | Working model of nitrate/nitrite antiport by NarK. The key residues in the coupling between the structural change and the substrate recognition (R89, Y263 and R305) and substrates (nitrate and nitrite) are shown. The TM helices that become bent during the transport cycle (TM7, TM10 and TM11) in the C bundle are illustrated as amber, pink and purple sticks, respectively, while those in the $\mathrm{N}$ bundle are represented by light blue sticks. The approximate locations of the important Gly residues for the TM bending are coloured green. The states corresponding to the present crystal structures are highlighted by red rectangles.

half of the transport cycle awaits the determination of the outward-open structure at atomic resolutions.

\section{Methods}

Expression and purification of NarK. The narK gene (GI: 945783) was cloned from the E. coli str. K-12 substr. MG1655 genome (ATCC 47076) using the NarK (WT) primers described in Supplementary Table 1, and subcloned into a pET modified vector ${ }^{28}$. The resultant plasmids were expressed in E. coli C41(DE3) $\triangle a c r B$ cells, and the proteins were purified as follows. The cells were grown in a $10-\mathrm{l}$ culture at $37^{\circ} \mathrm{C}$ to an absorbance at $600 \mathrm{~nm}$ of $0.4-0.6$, and expression was induced with $0.5 \mathrm{mM}$ IPTG at $20^{\circ} \mathrm{C}$ for $24 \mathrm{~h}$. The cells were then harvested using centrifugation $\left(4,500 \mathrm{~g}, 10 \mathrm{~min}, 4^{\circ} \mathrm{C}\right)$, resuspended in buffer containing $50 \mathrm{mM}$ Tris- $\mathrm{HCl}$ ( $\mathrm{pH} 8.0$ ), $300 \mathrm{mM} \mathrm{NaCl}, 20 \mathrm{mM} \mathrm{NaNO}_{3}, 0.1 \mathrm{mM}$ phenylmethylsulfonyl fluoride (PMSF) and $4 \mathrm{mM} \beta$-mercaptoethanol ( $\beta$-ME), and disrupted by three passages through a Microfluidizer processor (Microfluidics) at 15,000 p.s.i. After removal of the debris using centrifugation $\left(12,000 \mathrm{~g}, 30 \mathrm{~min}, 4^{\circ} \mathrm{C}\right)$, the supernatant was ultracentrifuged $\left(138,000 \mathrm{~g}, 1 \mathrm{~h}, 4^{\circ} \mathrm{C}\right)$ to pellet the membranes, which were then solubilized in a buffer containing $50 \mathrm{mM}$ Tris- $\mathrm{HCl}(\mathrm{pH} 8.0), 300 \mathrm{mM} \mathrm{NaCl}, 20 \mathrm{mM}$ $\mathrm{NaNO}_{3}, 0.1 \mathrm{mM}$ PMSF, $4 \mathrm{mM} \beta$-ME, $2 \%$ n-dodecyl- $\beta$-D-maltoside (DDM) and $0.4 \%$ cholesteryl hemisuccinate (CHS). Insoluble materials were removed using ultracentrifugation $\left(138,000 \mathrm{~g}, 30 \mathrm{~min}, 4^{\circ} \mathrm{C}\right)$, and the supernatant was mixed with $10 \mathrm{ml} \mathrm{Ni-NTA}$ Superflow resin (Qiagen). After binding for $2 \mathrm{~h}$, the resin was washed with $50 \mathrm{mM}$ Tris- $\mathrm{HCl}(\mathrm{pH} 8.0), 300 \mathrm{mM} \mathrm{NaCl}, 25 \mathrm{mM} \mathrm{NaNO}_{3}, 4 \mathrm{mM}$ $\beta$-ME, $0.05 \%$ DDM, $0.01 \%$ CHS and $20 \mathrm{mM}$ imidazole, and NarK was eluted with the same buffer supplemented with $300 \mathrm{mM}$ imidazole at $4{ }^{\circ} \mathrm{C}$. The GFP-His ${ }_{8}$ tag was cleaved by a His-tagged tobacco etch virus protease (produced in-house) and dialysed against the buffer without imidazole. The sample was reloaded on the 10-ml Ni-NTA column, to remove the protease. The flow-through fraction containing NarK was collected, concentrated and further purified on a HiLoad $16 / 600$ Superdex $200 \mathrm{pg}$ column (GE Healthcare), in $20 \mathrm{mM}$ Tris- $\mathrm{HCl}$ (pH 8.0), $150 \mathrm{mM} \mathrm{NaCl}, 25 \mathrm{mM} \mathrm{NaNO}_{3}, 4 \mathrm{mM} \beta$-ME, $0.05 \% \mathrm{DDM}$ and $0.01 \% \mathrm{CHS}$. The purified protein was concentrated to $15-20 \mathrm{mg} \mathrm{ml}^{-1}$ with a centrifugal filter device (Millipore, $50 \mathrm{kDa}$ molecular weight cutoff) and frozen until crystallization.

Crystallization. For the LCP crystallization trials, the protein solution was mixed with mono-olein (1-oleoyl-rac-glycerol) at a $4: 6 \mathrm{w} / \mathrm{w}$ ratio using coupled syringe devices, and the 30- to $80-\mathrm{nl}$ protein-laden LCP droplets were overlaid with $1 \mu \mathrm{l}$ precipitant solution on either 96-well plastic sandwich plates by the crystallization robot, mosquito LCP (TTP LabTech) or glass sandwich plates by the repeating dispenser (Hamilton). The crystals of NarK were grown at $20^{\circ} \mathrm{C}$, and three different types of crystals (I, II and III) were obtained in reservoir solutions I, II and III, respectively (reservoir solution I: 27\% PEG500DME, $100 \mathrm{mM}$ MES-NaOH (pH 6.5), $100 \mathrm{mM} \mathrm{NH}_{4} \mathrm{NO}_{3}$; reservoir solution II: $36 \%$ PEG400, $100 \mathrm{mM}$ MES-NaOH (pH 6.0), $10 \mathrm{mM} \mathrm{Zn(OAc)}$, $3 \%$ sucrose; reservoir solution III: $31 \%$ PEG500DME, $100 \mathrm{mM}$ MES-NaOH (pH 5.8), $100 \mathrm{mM} \mathrm{NH}_{4} \mathrm{NO}_{3}$ ). All crystals grew to full size in 2-3 weeks. The heavy atom-derivative crystals were prepared using the soaking method. After the native crystal III was grown on glass sandwich plates to the full size, $\sim 15 \times 40 \times 80 \mu \mathrm{m}$, the overlaid crystallization solution $(1 \mu \mathrm{l})$ was replaced by $1-1.5 \mu \mathrm{l}$ of the solution supplemented with a slightly lower concentration of PEG500DME and $4 \mathrm{mM} \mathrm{CH}_{3} \mathrm{HgCl}$. The crystal was incubated at $20^{\circ} \mathrm{C}$ for $5 \mathrm{~h}$ without drying. It was harvested using micromounts (MiTeGen), and was flash-cooled and stored in liquid nitrogen.

Data collection and structure determination. Diffraction data were collected at the SPring- 8 BL32XU beamline, using a $1 \mu \mathrm{m} \times 15 \mu \mathrm{m}$ (width-height) microbeam ${ }^{29}$. The data-processing statistics are summarized in Table 1 . The structure was determined using the single isomorphous replacement with anomalous scattering (SIRAS) method, using the native and $\mathrm{CH}_{3} \mathrm{HgCl}$-soaked crystals. Six $\mathrm{Hg}$ atom sites were identified with the programme SHELXD ${ }^{30,31}$. The initial phases were calculated using the programme SHARP ${ }^{32}$, followed by solventflattening with SOLOMON ${ }^{33}$ and twofold noncrystallographic symmetry averaged using $\mathrm{DM}^{34}$. The initial model structure of NarK was built with the programme Phyre2 (ref. 35), using the glycerol-3-phosphate transporter GlpT structure (PDB accession code: $1 \mathrm{PW} 4)^{36}$ as a template. The model was further built manually using $\mathrm{COOT}^{37,38}$ and refined using PHENIX ${ }^{39,40}$. Using this Crystal III structure as a search model, the Crystal I and II structures were determined with the molecular replacement method, using the programme PHASER ${ }^{41}$. Crystals I and II contain one molecule in each asymmetric unit, while Crystal III contains two molecules (Mol A and B) in each asymmetric unit. Mol A and B in Crystal III are essentially the same (r.m.s.d. of $0.38 \AA$ for all $\mathrm{C} \alpha$ atoms), and thus we only discussed $\mathrm{Mol} \mathrm{A}$ in the main text. The structure refinement statistics are summarized in Table 1.

MD simulations. We performed MD simulations starting from the apo occluded, nitrate-bound occluded and apo inward-open states. The present crystal structures of the nitrate-bound occluded and apo inward-open states were used as initial 
models. All of the lipid molecules observed in the crystal structures were removed, while all of the water molecules observed in the crystal structures were kept. The disordered loop connecting the $\mathrm{N}$ and $\mathrm{C}$ bundles was modelled by the programme MODELLER $^{42}$. For the apo-occluded simulation, the bound nitrate ion was removed from the crystal structure. The missing side chains and hydrogen atoms were built with the programme $\mathrm{VMD}^{43}$. The prepared structures were then embedded in a fully hydrated POPE bilayer ${ }^{4} 4$. In the crystal structure, several mono-olein molecules were observed around the TM segments, and these were used as indicators for the locations of the membrane. The lipid molecules overlapping the protein were removed, resulting in 320 POPE molecules included overall. The lipid-protein complex was then hydrated to form the $96 \AA \times 96 \AA \times 96 \AA$ simulation box. Sodium and chloride ions were then added to neutralize the system with a salt concentration of $150 \mathrm{mM}$. The molecular topologies and parameters from the CHARMM36 force-field parameters, with $\varphi, \psi$ cross-term map correction, were used ${ }^{44}$.

MD simulations were performed with the programme NAMD 2.8 (ref. 45). The systems were first energy minimized for 1,000 steps with fixed positions of the nonhydrogen protein atoms, and then for another 1,000 steps with $10 \mathrm{kcal} \mathrm{mol}^{-1}$ restraints for the nonhydrogen protein atoms. For the equilibration, the system was first subjected to a 100-ps simulation run with harmonic restraints on the nonhydrogen protein atoms in the NPAT ensemble, to relax the solute molecules, and then was further equilibrated by a 1-ns simulation run with harmonic restraints on the non-hydrogen protein atoms in the NPT ensemble. Finally, 250-ns MD simulations without any restraints were performed in the NPT ensemble for the production runs. In these simulations, constant pressure (1 atm) and temperature $(300 \mathrm{~K})$ were maintained using Langevin dynamics and a Langevin piston, respectively. The particle mesh Ewald method was employed for the calculation of the electrostatic interactions ${ }^{46}$. The equation of motion was integrated with a time step of $2 \mathrm{fs}$.

Liposome-based nitrite-flux assay. For the preparation of NarK-reconstituted proteoliposomes, soybean lipid (L- $\alpha$-phosphatidylcholine-type IV-S; Sigma) was dissolved in chloroform and evaporated at room temperature. The lipid film deposited on the interior of a plastic tube was resuspended to a final concentration of $10 \mathrm{mg} \mathrm{ml}^{-1}$ in extraliposome buffer, containing $25 \mathrm{mM}$ HEPES- $\mathrm{NaOH}(\mathrm{pH}$ 7.0), and $50 \mathrm{mM}$ of either $\mathrm{NaNO}_{3}, \mathrm{NaCl}, \mathrm{NaHCO}_{3}, \mathrm{HCOONa}, \mathrm{CH}_{3} \mathrm{COONa}$ or $\mathrm{CH}_{3} \mathrm{CH}_{2} \mathrm{COONa}$. The suspension was sonicated for $30 \mathrm{~s}$ to form unilamellar vesicles. Purified proteins were reconstituted into liposomes, at a lipid/protein ratio of 100:1 (w/w), by three rounds of freezing and thawing. The proteoliposomes were centrifuged at $210,000 \mathrm{~g}$ for $30 \mathrm{~min}$. The nitrite-uptake reaction was initiated by resuspending the pellet in intraliposome buffer, containing $25 \mathrm{mM}$ HEPES-NaOH (pH 7.0) and $50 \mathrm{mM} \mathrm{NaNO}_{2}$. After an incubation at room temperature for $1 \mathrm{~h}$, the reaction mixture was loaded on a Sephadex G-50 column $(0.5 \times 4.0 \mathrm{~cm})$ equilibrated with $25 \mathrm{mM}$ HEPES- $\mathrm{NaOH}$ ( $\mathrm{pH}$ 7.0). The eluted fraction was mixed with sodium dodecyl sulfate, to disrupt the liposomes, and then with $\mathrm{HCl}$, in order to lower the $\mathrm{pH}$, to final concentrations of $0.5 \%$ and $0.1 \mathrm{M}$, respectively. The solution was mixed with DAF-FM ${ }^{23}$ and incubated at room temperature for $2 \mathrm{~min}$. The solution was diluted 10 -fold with $25 \mathrm{mM}$ HEPES- $\mathrm{NaOH}(\mathrm{pH} 7.0)$ and incubated at room temperature for $2 \mathrm{~min}$. The fluorescence of triazolofluorescein was monitored with excitation at $500 \mathrm{~nm}$ and emission detected at $515 \mathrm{~nm}$, using an F-7000 fluorescence spectrophotometer (Hitachi). To generate the membrane potential in proteoliposomes, we added $10 \mathrm{mM} \mathrm{KCl}$ to the intraliposome buffer, and $100 \mathrm{mM}$ $\mathrm{KCl}$ with $10 \mu \mathrm{M}$ valinomycin to the extraliposome buffer. To change the $\mathrm{pH}$ of the intra- and extraliposome buffers, MES- $\mathrm{NaOH}(\mathrm{pH}$ 6.0) and Tris- $\mathrm{HCl}(\mathrm{pH} 8.0)$ were used.

Genetic analysis of the transport activity of NarK mutants. The genetic analysis of the NarK mutants was performed essentially as described previously ${ }^{15}$. In brief, in the presence of TMAO under anaerobic conditions, E. coli assimilates TMAO, which is reduced to the strong base trimethylamine by endogenous TMAO reductases, resulting in the formation of pale yellow spots on MacConley-glucoseTMAO agar plates. In contrast, if nitrate is assimilated by E. coli cells during anaerobic growth, it prevents TMAO reduction by out-competing TMAO reductase for electrons from the quinol pool ${ }^{15}$, resulting in the formation of dark red spots. The E. coli strain constructed for this assay lacks all of the endogenous nitrate/nitrite transporters and channels (NarK, NarU and NirC). Therefore, the colours of the cells grown anaerobically in the presence of both nitrate and TMAO completely depend on the nitrate-uptake activity of the plasmid-encoded NarK.

To construct the assay strain with the sextuplet gene knockout ( $\triangle$ napA-napB, $\Delta$ narZ-narU, $\Delta$ nirC, $\Delta$ narK), each gene's knockout allele was constructed and transferred step-by-step to the destination strain BW25113, principally by the method described in ref. 47, using appropriate PCR primers (NAP1 and NAP2 for $\Delta$ napA-napB, NAR1 and NAR2 for $\Delta$ narZ-narU; Supplementary Table 2) or P1 phage transduction from a systematic E. coli knockout library strain $(\Delta$ nirC, $\Delta$ narK $)^{48}$. We isolated $\lambda$ DE3 lysogenic strains, which carries the inducible T7 polymerase system for the gene expression ${ }^{49}$, by spotting $\lambda$ DE3 lysate together with appropriate helper phage lysate on the host strain. Under the noninduced conditions in the absence of lactose or IPTG, the assay strain expresses a stable amount of T7 polymerase, which is sufficient for the assays of NarK mutants with severe phenotypes ('no-induction system'; Fig. 3c). In contrast, to evaluate the nitrate transport activity of NarK and its mutants depending on their expression levels, another expression system was constructed. In the presence of pLysS, the T7 polymerase activity is reduced, thus allowing more stringent control of expression from the T7 promoter, depending on the IPTG concentration ('inducible system'). We used this inducible system under three different conditions (IPTG $=0,23$ and $67 \mu \mathrm{M}$ ) for the assays of mutants involved in substrate recognition (Fig. 5c).

Transformants harbouring the WT or mutant narK gene derived from the exogenous plasmid were cultured overnight at $37^{\circ} \mathrm{C}$ in liquid LB medium, supplemented with appropriate antibiotics, diluted 50 -fold in the same medium and then grown to an optical density (OD) 600 of 0.6 at $37^{\circ} \mathrm{C}$. The refreshed log-phase cultures were spotted on modified MacConkey-glucose-nitrate-TMAO plates $^{50}$, containing $0.5 \mathrm{~g} \mathrm{KNO}_{3}, 0.8 \mathrm{~g}$ D-glucose and $2.0 \mathrm{~g}$ TMAO in $200 \mathrm{ml}$ MacConkey agar medium, and grown anaerobically at $30^{\circ} \mathrm{C}$ for $12 \mathrm{~h}$ using AnaeroPack jar systems (AnaeroPack Kenki 5\% A-07, Mitsubishi Gas Chemical Company Inc., Tokyo, Japan).

\section{References}

1. Martínez-Espinosa, R. M., Cole, J. A., Richardson, D. J. \& Watmough, N. J. Enzymology and ecology of the nitrogen cycle. Biochem. Soc. Trans. 39, 175-178 (2011).

2. Dixon, R. \& Kahn, D. Genetic regulation of biological nitrogen fixation. Nat. Rev. Microbiol. 2, 621-631 (2004).

3. Stewart, V. Nitrate respiration in relation to facultative metabolism in enterobacteria. Microbiol. Rev. 52, 190-232 (1988).

4. Nacry, P., Bouguyon, E. \& Gojon, A. Nitrogen acquisition by roots: physiological and developmental mechanisms ensuring plant adaptation to a fluctuating resource. Plant Soil. 370, 1-29 (2013).

5. Omata, T. Structure, function and regulation of the nitrate transport system of the cyanobacterium Synechococcus sp. PCC7942. Plant Cell Physiol. 36, 207-213 (1995).

6. Tsay, Y. F., Chiu, C. C., Tsai, C. B., Ho, C. H. \& Hsu, P. K. Nitrate transporters and peptide transporters. FEBS Lett. 581, 2290-2300 (2007).

7. Léran, S. et al. A unified nomenclature of NITRATE TRANSPORTER 1/PEPTIDE TRANSPORTER family members in plants. Trends Plant Sci. 19, 5-9 (2014)

8. MacGregor, B. J., Biddle, J. F., Harbort, C., Matthysse, A. G. \& Teske, A. Sulfide oxidation, nitrate respiration, carbon acquisition, and electron transport pathways suggested by the draft genome of a single orange Guaymas Basin Beggiatoa (Cand. Maribeggiatoa) sp. filament. Mar. Genomics 11, 53-65 (2013)

9. Pao, S. S., Paulsen, I. T. \& Saier, M. H. Major facilitator superfamily. Microbiol. Mol. Biol. Rev. 62, 1-34 (1998).

10. Forde, B. G. Nitrate transporters in plants: structure, function and regulation Biochim. Biophys. Acta 1465, 219-235 (2000).

11. Rowe, J. J., Ubbink-Kok, T., Molenaar, D., Konings, W. N. \& Driessen, A. J. M. NarK is a nitrite-extrusion system involved in anaerobic nitrate respiration by Escherichia coli. Mol. Microbiol. 12, 579-586 (1994).

12. Clegg, S. J., Jia, W. \& Cole, J. A. Role of the Escherichia coli nitrate transport protein, NarU, in survival during severe nutrient starvation and slow growth. Microbiology 152, 2091-2100 (2006).

13. Noji, S., Nohno, T., Saito, T. \& Taniguchi, S. The narK gene product participates in nitrate transport induced in Escherichia coli nitrate-respiring cells. FEBS Lett. 252, 139-143 (1989).

14. DeMoss, J. A. \& Hsu, P. Y. NarK enhances nitrate uptake and nitrite excretion in Escherichia coli. J. Bacteriol. 173, 3303-3310 (1991).

15. Clegg, S., Yu, F., Griffiths, L. \& Cole, J. A. The roles of the polytopic membrane proteins NarK, NarU and NirC in Escherichia coli K-12: two nitrate and three nitrite transporters. Mol. Microbiol. 44, 143-155 (2002).

16. Jia, W., Tovell, N., Clegg, S., Trimmer, M. \& Cole, J. A single channel for nitrate uptake, nitrite export and nitrite uptake by Escherichia coli NarU and a role for NirC in nitrite export and uptake. Biochem. J. 417, 297-304 (2009).

17. Moir, J. W. \& Wood, N. J. Nitrate and nitrite transport in bacteria. Cell. Mol. Life Sci. 58, 215-224 (2001).

18. Yan, H. et al. Structure and mechanism of a nitrate transporter. Cell Rep. 3, 716-723 (2013).

19. Zheng, H., Wisedchaisri, G. \& Gonen, T. Crystal structure of a nitrate/nitrite exchanger. Nature 497, 647-651 (2013).

20. Radestock, S. \& Forrest, L. R. The alternating-access mechanism of MFS transporters arises from inverted-topology repeats. J. Mol. Biol. 407, 698-715 (2011).

21. Parker, J. L. \& Newstead, S. Molecular basis of nitrate uptake by the plant nitrate transporter NRT1.1. Nature 507, 68-72 (2014).

22. Sun, J. et al. Crystal structure of the plant dual-affinity nitrate transporter NRT1.1. Nature 507, 73-77 (2014)

23. Kojima, H. et al. Detection and imaging of nitric oxide with novel fluorescent indicators: diaminofluoresceins. Anal. Chem. 70, 2446-2453 (1998).

24. Landau, E. M. \& Rosenbusch, J. P. Lipidic cubic phases: a novel concept for the crystallization of membrane proteins. Proc. Natl Acad. Sci. USA 93, 14532-14535 (1996). 
25. Trueman, L. J., Richardson, A. \& Forde, B. G. Molecular cloning of higher plant homologues of the high-affinity nitrate transporters of Chlamydomonas reinhardtii and Aspergillus nidulans. Gene 175, 223-231 (1996).

26. Unkles, S. E. et al. Two perfectly conserved arginine residues are required for substrate binding in a high-affinity nitrate transporter. Proc. Natl Acad. Sci. USA 101, 17549-17554 (2004).

27. Fischer, K. et al. Structural basis of eukaryotic nitrate reduction: crystal structures of the nitrate reductase active site. Plant Cell 17, 1167-1179 (2005).

28. Nishizawa, T. et al. Structural basis for the counter-transport mechanism of a $\mathrm{H}^{+} / \mathrm{Ca}^{2+}$ exchanger. Science 341, 168-172 (2013).

29. Hirata, K. et al. Achievement of protein micro-crystallography at SPring-8 beamline BL32XU. J. Phys. Conf. Ser. 425, 12002 (2013).

30. Schneider, T. R. \& Sheldrick, G. M. Substructure solution with SHELXD. Acta Crystallogr. D Biol. Crystallogr. 58, 1772-1779 (2002).

31. Sheldrick, G. M. A short history of SHELX. Acta Crystallogr. A 64, 112-122 (2008).

32. de La Fortelle, E., Irwin, J. \& Bricogne, G. SHARP: a maximum-likelihood heavy-atom parameter refinement program for the MIR and MAD methods. Methods Enzym 276, 472-494 (1997).

33. Abrahams, J. P. \& Leslie, A. G. Methods used in the structure determination of bovine mitochondrial F1 ATPase. Acta Crystallogr. D Biol. Crystallogr. 52, 30-42 (1996).

34. Cowtan, K. D. \& Main, P. Improvement of macromolecular electron-density maps by the simultaneous application of real and reciprocal space constraints. Acta Crystallogr. D Biol. Crystallogr. 49, 148-157 (1993).

35. Kelley, L. A. \& Sternberg, M. J. E. Protein structure prediction on the Web: a case study using the Phyre server. Nat. Protoc. 4, 363-371 (2009).

36. Huang, Y., Lemieux, M. J., Song, J., Auer, M. \& Wang, D. N. Structure and mechanism of the glycerol-3-phosphate transporter from Escherichia coli. Science 301, 616-620 (2003).

37. Emsley, P. \& Cowtan, K. Coot: model-building tools for molecular graphics. Acta Crystallogr. D Biol. Crystallogr. 60, 2126-2132 (2004).

38. Emsley, P., Lohkamp, B., Scott, W. G. \& Cowtan, K. Features and development of Coot. Acta Crystallogr. D Biol. Crystallogr. 66, 486-501 (2010).

39. Adams, P. D. et al. PHENIX: building new software for automated crystallographic structure determination. Acta Crystallogr. D Biol. Crystallogr. 58, 1948-1954 (2002).

40. Adams, P. D. et al. PHENIX: a comprehensive Python-based system for macromolecular structure solution. Acta Crystallogr. D Biol. Crystallogr. 66, 213-221 (2010).

41. McCoy, A. J. et al. Phaser crystallographic software. J. Appl. Crystallogr. 40, 658-674 (2007)

42. Sali, A., Potterton, L., Yuan, F., van Vlijmen, H. \& Karplus, M. Evaluation of comparative protein modeling by MODELLER. Proteins 23, 318-326 (1995).

43. Humphrey, W., Dalke, A. \& Schulten, K. VMD: visual molecular dynamics. J. Mol. Graph. 14, 27-28 (1996).

44. Klauda, J. B. et al. Update of the CHARMM all-atom additive force field for lipids: validation on six lipid types. J. Phys. Chem. B 114, 7830-7843 (2010).

45. Phillips, J. C. et al. Scalable molecular dynamics with NAMD. J. Comput. Chem. 26, 1781-1802 (2005).

46. Darden, T., York, D. \& Pedersen, L. Particle mesh Ewald: an N $\cdot \log (\mathrm{N})$ method for Ewald sums in large systems. J. Chem. Phys. 98, 10089 (1993).

47. Datsenko, K. A. \& Wanner, B. L. One-step inactivation of chromosomal genes in Escherichia coli K-12 using PCR products. Proc. Natl Acad. Sci. USA 97, 6640-6645 (2000).

48. Baba, T. et al. Construction of Escherichia coli $\mathrm{K}-12$ in-frame, single-gene knockout mutants: the Keio collection. Mol. Syst. Biol. 22006.0008 (2006).
49. Studier, F. W., Rosenberg, A. H., Dunn, J. J. \& Dubendorff, J. W. Use of T7 RNA polymerase to direct expression of cloned genes. Methods Enzymol. 185, 60-89 (1990).

50. Stewart, V. \& MacGregor, C. H. Nitrate reductase in Escherichia coli K-12: involvement of chlC, chlE, and chlG loci. J. Bacteriol. 151, 788-799 (1982).

\section{Acknowledgements}

We thank M. Hattori (University of Tokyo, Japan), T. Nishizawa (University of Tokyo), H. Kojima (University of Tokyo) and S. Newstead (University of Oxford, UK) for useful discussions and critical comments on the manuscript; T. Tsukazaki (University of Tokyo) for sharing beamtime; A. Kurabayashi (University of Tokyo) for technical assistance; K. Hirata, the beam-line staff at BL32XU of SPring-8 (Hyogo, Japan) for assistance in data collection; and the RIKEN BioResource Center (Ibaraki, Japan) for providing the E. coli str. K-12 substr. MG1655 genomic DNA. The diffraction experiments were performed at SPring-8 BL32XU (proposals 2012A1093, 2012B1146, $2012 \mathrm{~B} 1162$ and 2013A1168) and with the approval of RIKEN. The computations were performed using mini-K super computer system at the SACLA facility. This work was supported by the Japan Society for the Promotion of Science (JSPS) through its 'Funding Program for World-Leading Innovative R\&D on Science and Technology (FIRST program)' to O.N., by the Core Research for Evolutional Science and Technology (CREST) Program 'The Creation of Basic Medical Technologies to Clarify and Control the Mechanisms Underlying Chronic Inflammation' of Japan Science and Technology Agency (JST) to O.N., by a grant for HPCI STRATEGIC PROGRAM Computational Life Science and Application in Drug Discovery and Medical Development by MEXT to R.I. and by a Grant-in-Aid for Scientific Research (S) (24227004) and a Grant-in-Aid for Young Scientists (A) (22687007) from the MEXT to O.N. and R.I., respectively.

\section{Author contributions}

M.F. expressed, purified and crystallized NarK, and collected the diffraction data and solved the structures. M.F. made the mutants for genetic analyses of the nitrate transport activity of NarK. H.T. and M.F. performed in vitro assays using proteoliposomes. K.I. constructed the gene knockout E. coli strains and performed genetic analyses. S.D. purified proteins for in vitro assays. R.I. performed MD simulations. A.D.M. performed experiments using fluorescent probes before the liposome-based nitrite-flux assay. M.F. H.E.K., K.I., R.I. and O.N. designed the research; M.F., H.E.K., R.I. and O.N. wrote the manuscript. R.I. and O.N. directed and supervised all of the research.

\section{Additional information}

Accession codes. The coordinates and the structure factors have been deposited in the Protein Data Bank (PDB), under the accession codes 4U4V (apo inward-open state), 4U4T (nitrate-bound inward-open state) and 4U4W (nitrate-bound occluded state).

Supplementary Information accompanies this paper at http://www.nature.com/ naturecommunications

Competing financial interests: The authors declare no competing financial interests

Reprints and permission information is available online at http://npg.nature.com/ reprintsandpermissions/

How to cite this article: Fukuda, M. et al. Structural basis for dynamic mechanism of nitrate/nitrite antiport by NarK. Nat. Commun. 6:7097 doi: 10.1038/ncomms8097 (2015)

This work is licensed under a Creative Commons Attribution 4.0 International License. The images or other third party material in this article are included in the article's Creative Commons license, unless indicated otherwise in the credit line; if the material is not included under the Creative Commons license, users will need to obtain permission from the license holder to reproduce the material. To view a copy of this license, visit http://creativecommons.org/licenses/by/4.0/ 